\title{
DEVELOPMENT OF AN ADVANCED ATMOSPHERIC/TRANSPORT MODEL FOR EMERGENCY RESPONSE PURPOSES (U)
}

by

J. D. Fast

Westinghouse Savannah River Company

Savannah River Site

Aiken, South Carolina 29808

A Technical Report for publication on

July 12,1991

This paper was prepared in connection with work done under Contract No. DE-AC09-89SR18035 with the U.S. Department of Energy. By acceptance of this paper, the publisher and/or recipient acknowledges the U.S. Government's right to retain a nonexclusive, royalty-free license in and to any copyright covering this paper, along with the right to reproduce and to authorize others to reproduce all or part of the copyrighted paper. 
WSRC-RP-91-577

Retention: 2 years

\section{DEVELOPMENT OF AN ADVANCED ATMOSPHERIC/ TRANSPORT MODEL FOR EMERGENCY RESPONSE PIURPOSES (U)}

\author{
J. D. FAST \\ B. L. O'STEEN \\ R. P. ADDIS
}

Publication Date: May 3, 1991

\section{DISCLAIMER}

This report was prepared as an account of work sponsored by an agency of the United States This report was prepared as an account of work Government. Neither the United States Government nor any agency thereof, nor any of their employees, makes any warranty, express or implied, or assumes any legal liability or reduct, or bility for the accuracy, completeness, or usefulness of any information, apparatus, product, or process disclosed, or represents that its use would not infringe privately owned rights. Reference herein to any specific commercial product, process, or service by trade name, tradentark, manufacturer, or otherwise does not necessarily constitute or imply its endorsement, recommanufacturer, or otherwise died States Government or any agency thereof. The views mendations of authors expressed herein do not necessarily state or reflect those of the United States Government or any agency thereof.

Westinghouse Savannah River Company

Savannah River Site

Aiken, SC 29808 
CONTENTS

INTRODUCTION

GOAL

APPROACH 2

1.0 Site-Specific Characteristics and Parameterizations 3

2.0 Boundary Conditions $\quad 5$

3.0 Initialization and Data Assimilation $\quad 6$

4.0 Dispersion and Dosimetry $\quad 7$

$\begin{array}{llr}5.0 & \text { Operational Structure and System Integration } & 8\end{array}$

TARGET DATES 9

CONCLUSION

FIGURES 1 - 2 12 


\title{
DEVELOPMENT OF AN ADVANCED ATMOSPHERIC/TRANSPORT MODEL FOR EMERGENCY RESPONSE PURPOSES
}

\author{
By J. D. Fast, B. L. O'Steen, and R. P. Addis \\ Westinghouse Savannah River Company \\ Savannah River Site \\ Aiken, SC 29808
}

\section{INTRODUCTION}

Atmospheric transport and diffusion models have been developed for real-time calculations of the location and concentration of toxic or radioactive materials during an accidental release at the Savannah River Site (SRS). These models are based on Gaussian distributions and have been incorporated into an automated menu-driven program called the WIND (Weather INformation and Display) system.

The WIND system atmospheric models employ certain assumptions that allow the computations of the ground-level concentration of toxic or radioactive materials to be made quickly Gaussian models, such as PF/PL and 2DPUF, suffer from serious limitations including the inability to represent recirculation of pollutants in complex terrain, the use of one stability class at a given time to represent turbuient mixing over heterogeneous terrain, and the use of a wind field computed at only one height in the atmosphere. These limitations arise because the fundamental conservation relations of the atmosphere have been grossly simplified.

Three-dimensional coupled atmospheric-dispersion models are not limited by the oversimplifications of the Gaussian assumption and have been used in the past to predict the transport of pollutants in a variety of atmospheric circulations. The disadvantage of these models is that they require large amounts of computational time; however, technology has progressed enough so that real-time simulations of dispersion may be made. These complex models can be run in an operational mode so that routine forecasts of the wind field and particulate concentration can be made.

\section{GOALS}

The Environment Transport Group (ETG) has recently acquired a prognostic three-dimensional mesoscale atmospheric model called RAMS (Regional Atmospheric Modeling System) from Colorado State University that simulates circulations with horizontal atmospheric scales ranging from 0.1 to $100 \mathrm{~km}$. A transport model called LPDM (Lagrangian Particle Dispersion Model) that incorporates the wind fields produced by RAMS has also been obtained. The RAMS and LPDM models will be coupled to a graphical packages, such as NCAR (National Center for Atmospheric Research) graphics. These models have been purchased with two goals in mind:

1. To develop a version of the RAMS and LPDM models which can produce forecasts in an emergency response capacity. This model would then be used to simulate the dispersion of toxic or radioactive materials by atmospheric circulations over the SRS area in emergency response situations (see Fig. 1).

2. A strictly research-oriented version of the RAMS and LPDM models will be used to simulate various atmospheric circulations over the SRS area. 
The research-oriented version of the model will be necessary to test new parameterizations and numerical techniques so that they can be incorporated into the operational (emergency response) version of the model.

\section{APPROACH}

Numerous tasks must be completed to achieve the goals of the project. These tasks can be grouped into five broad areas of research and development which are:

1. Site-Specific Characteristics and Parameterizations: Determining the ability of the RAMS model to correctly simulate mesoscale atmospheric behavior in the SRS area, given that the synoptic state is imposed correctly.

2. Boundary Conditions: Interfacing the RAMS model with larger-scale NMC (National Meteorological Center) models to obtain lateral boundary conditions. The problem of realtime data retrieval will have to be addressed.

3. Initialization and Data Assimilation: Assimilating observed data from NMC and WCAL (Weather Center Atmospheric Laboratory) into the RAMS model to obtain improved wind-field forecasts. The problem of real-time data retrieval and executing the model in an operational mode needs to be examined.

4. Dispersion and Dosimetry: Coupling the RAMS model to the LPDM model and calculating concentration and dose estimates.

5. Operational Structure and System Integration: Determining the stability, accuracy, robustness of the RAMS and L.PDM codes and graphically depicting the concentration and dose estimates in an operational mode. The problem of reducing the overall computational time will have to be addressed.

A large portion of the research needed to complete this project will be performed by external research scientists, such as those associated with the University of South Carolina and Colorado State University through the South Carolina Universities and Research Foundation (SCUREF). It is anticipated that important contributions will also be made by other companies that developed the RAMS and LPDM codes including Atmospheric Simulation Testing and Research Corporation (ASTeR) and Supercomputer Systems Engineering and Services Company (SSESCO) (see Fig. 2).

Each of the areas of research and development can be divided into several subtasks. It is expected the the completion of every essential task in this project will take approximately four years. Nevertheless, a simplified preliminary operation version the advanced atmospheric/transport modeling system should be ready by the summer of 1992. A list of the detailed tasks, along with the researchers who will complete those tasks and the projected completion dates, are included below. It is anticipated that some of the subtasks will not be completed by the target date; however, progress towards completing the major task should be documented at that time. 


\subsection{Site Specific Characteristics and Parameterizations}

1.1 Familiarization: The specific governing equations, parameterizations, and numerical procedures used by the RAMS code needs to be examined. All of the options available from the RAMS code that are particularly suited to simulating atmospheric circulations over the SRS area need to be identified. The particular characteristics of the LPDM dispersion model and the graphical packages will also need to be examined.

\section{Lance O'Steen, Jerome Fast}

(on-going)

1.2 Documentation: Portions of the RAMS code have been described in many meteorological journal articles; however, a single source that lists all of the governing equations, parameterizations, and numerical methods used by the current version of the model should be written.

ASTeR, Lance O'Steen, Jerome Fast

1.2.1 A document should be written that contains the current governing equations, parameterizations, and numerical represuntations used by RAMS and LPDM codes.

1.2.2 After each major task has been completed and incorporated into the modeling system, any modifications to the RAMS code that was necessary for the SRS application should be thoroughly documented.

1.2.3 The RAMS and LPDM models should be evaluated to demonstrate that it is capable of realistically simulating the dispersion of toxic chemicals or radionuclides in the SRS area. This can be done by executing the most current versions of RAMS and LPDM to simulate dispersion from observational tracer experiments such as MATS (Mesoscale Atmospheric Tracer Studies) and ASCOT (Atmospheric Studies in Complex Terrain). Past performance studies of the RAMS and LPDM models should also be referenced.

1.2.4 Once the model is ready to be implemented in to the WIND system, quality assurance documentation on the software will have to be written.

1.2.5 A number of journal articles should be written to present to the scientific community the results of the development and implementation of an advanced atmosopheric/tranport modeling system at SRS. Articles should be submitted to meteorological, environmental, nuclear, and computational publications.

1.3 Grid Structure: The size of the domain, grid spacing, and number of nested grids that will be adequate to simulate the atmospheric circulations over the SRS area must be determined.

Jerome Fast

1.3.1 The domain size necessary for simulating dispersion in the SRS area will need to be determined.

1.3.2 The number of nested grids within the domain must be chosen, based partially on computational requirements.

1.3.3 The horizontal and vertical grid spacing necessary to resolve the atmospheric circulations within the chosen domain must be determined.

1.3.4 The model code needs to be checked or modified to ensure that the nested grids use actual surface characteristics, such as topography, rather than interpolated values from the next largest grid.

1.3.5 The simulation period needs to be defined for emergency response situations. 
1.3.6 A simulation should be performed to determine how much computational time is necessary for the domain and the number of nested grids chosen in task 1.2.

1.3.7 RAMS is currently being executed on a Cray XMP computer. The model will eventually have to be compiled and executed on a workstation (an IBM RS/6000 workstation is currently being purchased) so that there will be no competing processes that might increase the total execution time. A comparison of the computational times required by each machine should be made.

1.4 Parameterization: Certain parameterizations of the RAMS code may be more appropriate for the SRS application. These parameterizations must execute with sufficient speed to be useful in emergency response situations. Additional parameterizations may need to be incorporated into RAMS to simulate the atmospheric circulations in the SRS area with a higher degree of accuracy.

Jerome Fast, Roger Pielke, ASTER

1.4.1 The RAMS code may have several different options for the parameterization of one physical process (such as turbulence). These options must be evaluated to determine which ones are more applicable to the SRS area. The criteria for this task will be based only on theoretical and computational factors.

1.4.2 The parameterizations chosen in task 1.4.1 should be employed in a one-dimensional version of RAMS. The numerical results should be compared with observational data taken from the WCAL data base for relatively stationary synoptic flow patterns to assess the performance of the model and the parameterizations. The ETS sodar system and the WJBF television tower can be used to evaluate the predicted vertical profiles of temperature and wind and the ETS sodar system can be used to evaluate the predicted boundary-layer height.

1.4.3 The parameterizations chosen in task 1.4.1 should be employed in a three-dimensional version of RAMS. The numerical results should be compared with observational data taken from the WCAL data base for relatively stationary synoptic flow patterns to assess the performance of the model. The ETS meteorological towers can be used to evaluate the predicted horizontal wind field.

1.4.4 For emergency response purposes, it is economical to execute RAMS without the gridscale condensation, latent heat release, precipitation, or cumulus convection parameterizations; however, there will be many times when these processes are important factors in influencing the small-scale circulations in the boundary-layer for the SRS application. The RAMS code will have to be tested to determine whether these parameterizations need to be used.

1.4.5 To more accurately predict the thermal and dynamic fields near the surface, a vegetation parameterization should eventually be incorporated into the model. A vegetation parameterization will be an option included in a future version of RAMS and it will have to be evaluated for the SRS application.

1.4.6 To more accurately predict the turbulence characteristics in the boundary-layer, secondorder closure techniques should be tested using RAMS. Second-order closure techniques require more computational time; therefore, the predicted flow fields and turbulence characteristics must be significantly superior to those predicted by first-order closure methods. A second-order closure technique will be an option included in a future version of RAMS and it will have to be evaluated for the SRS application.

1.4.7 Test simulations of RAMS must be performed for the SRS area when additional parameterizations are incorporated into the code. The parameterizations will be used if the resulting circulations agree with observed flows with a higher degree of accuracy that those produced in tasks 1.4.2 and 1.4.3. 


\subsection{Boundary Conditions}

2.1 Lateral Boundary Conditions: The RAMS model must be evaluated to determine which lateral boundary condition options perform the best when simulating atmospheric circulations in the SRS area. Methods of specifying the atmospheric variables at the lateral boundaries from larger scale, NMC models will have to be examined and developed.

Shun Der Ko, Chris Evans, Jerome Fast, ASTeR

2.1.1 The lateral boundary conditions can be provided by number of different methods. Constant, zero-gradient, and radiation boundary conditions are the simplest methods currently employed by RAMS. Each of these methods need to be evaluated to determine how useful they may be in a regional simulation over the SRS area.

2.1.2 The RAMS model also permits time-tendencies of known atmospheric variables to be used at the lateral boundary conditions; however, this method may need to be refined. These time-tendencies can be supplied by using values of atmospheric variables from objective analysis fields or NMC forecast models. Using forecasts from larger-scale models permits real-time mesoscale simulations to be made by RAMS; however, the numerical results will be affected by the errors in the larger-scale model. Using a series of analysis fields will greatly reduce the numerical errors generated at the lateral boundaries, but this technique could only be used in diagnostic studies to determine the errors produced be this technique.

2.1.3 A procedure will have to be developed to automatically access and archive NMC data (observations and forecasts). This would require additional hardware and software development. A direct link to NMC would also have to be established either by satellite or phone line. This data could also be obtained indirectly from a vendor. The amount of memory required for archiving data can be greatly reduced if old data no longer needed for mesoscale simulations are automatically purged. Data for special weather events, such as severe storms, could be stored manually.

2.1.4 A program would be necessary to read NMC data and interpolate it to the RAMS grids. ASTeR has a program that does this; however, additional software development may be necessary.

2.1.5 Currently, RAMS can read only two data files for the lateral boundary conditions. This may be sufficient if only a 12 hour simulation is required. However, if longer simulation periods are necessary, the code will have to be altered to read and interpolate more data files.

2.1.6 RAMS will be executed with no synoptic flow (with and without nested grids) to examine the thermally-induced circulations caused by inhomogeneous surface characteristics.

2.1.7 RAMS will be executed with observed synoptic flow pattern and present lateral and top boundary conditions (with and without nested grids) to examine the effect of synoptic flow on small-scale circulation patterns in the SRS area.

2.1.8 RAMS will be executed with observed synoptic flow pattern and observed synoptic tendencies at lateral and top boundaries (with and without nested grids) to exanine the effect of boundary conditions on the interior flow.

2.1.9 Test runs will be performed to evaluate the numerical noise generated by the timedependent lateral boundary conditions.

2.2 Top Boundary Conditions: The top boundary conditions available from RAMS are adequate when simulating atmospheric circulations in the SRS area; however, if computational restrictions limit the vertical extent of the domain, modifications to the code may be necessary.

Jerome Fast, Lance O'Steen 
2.2.1 The top boundary values for all prognostic variables in RAMS are extrapolated from interior, except for pressure and vertical velocity which incorporate a radiation-type boundary condition to suppress the reflection of gravity waves. This requires the model top to be set in the lower stratosphere at least $15 \mathrm{~km}$ above the surface. The model top may be set lower in the atmosphere to reduce the number of computations so that the emergency response version can execute faster. If this is necessary, the appropriateness of the current top boundary conditions will have to be examined.

2.2.2 Time-dependent top boundary conditions may also be used at the model top to drive the synoptic flow field. If this is chosen, data from NMC models will have to be accessed and archived so that it can be read into the RAMS model. A interpolation procedure would have to be disveloped, similar to the one used by the time-dependent lateral boundary conditions, so that the synoptic field could vary gradually in time. The question of how the time-dependent prognostic variables at the model top can be used when a radiation top boundary condition has been imposed will have to be addressed.

\subsection{Initialization and Data Assimilation}

3.1 Initial Conditions: The current horizontally homogeneous or inhomogeneous initial conditions must be tested to determine whether they are satisfactory for the SRS application. Additional modifications to these schemes may need to be performed.

Shun Der Ko, Chris Evans, Jerome Fast

3.1.1 RAMS currently reads in certain surface characteristics such as topography, percent land, soil type, and roughness for the model domain. Default roughness and soil type files need to be created for the domain to be used in the SRS application.

3.1.2 The method of determining horizontally inhomogeneous soil-moisture in the domain needs to be determined. RAMS currently assumes that a given soil-moisture profile is constant throughout the domain. The initial vertical profile of soil moisture will also have to be estimated. In the operational version of RAMS, the initial soil-moisture distribution would change for each initialization period.

3.1.3 The variable initial conditions section of the RAMS code needs to be evaluated. Horizontally homogeneous initial conditions are the simplest method in the model; however, they probably will not be accurate enough if the domain size is relatively large (mesoscale).

3.1.4 The specific initial conditions to be used for the emergency response application needs to be determined. The NMC analysis can be interpolated to the RAMS grid for the initial conditions; however, additional data from the WCAL data base may also need to be assimilated.

3.1.5 Other data assimilation techniques for the initial conditions, such as normal model initialization, will need to be examined to determine whether they are nomical or necessary for the SRS application.

3.1.6 Several simulations need to be made to determine the numerical noise and gravity waves generated by unbalanced initial conditions. Additional modifications to the variable initial conditions module of RAMS may need to be made to obtain an balanced initial field.

3.2 Four-Dimensional Data Assimilation: This task would involve the addition of another subroutine in the RAMS code that would assimilate observational data from NWS (National Weather Service) stations and the WCAL data base into the forecasts made by the RAMS model.

Jerome Fast, Shun Der Ko, Chris Evans, Roger Pielke 
3.2.1 Part of the four-dimensional data assimilation development involves the incorporation of time-tendencies at the lateral and top boundaries. In addition, NMC data and the WCAL data base should be used to improve numerical forecasts by the mesoscale model.

3.2.2 The type of data assimilation technique to be used in RAMS needs to be established. The most prevalent and economical method at present is the Newtonian nudging technique. This requires adding an extra term to each of the prognostic equations that will force the predicted value to the observed value. The prognostic equations can either nudge to an analysis of the larger- scale data or to the data at the station itself. Previous investigations have recommended that nudging should proceed towards that values at the stations for smaller-scale mesoscale models. The weighting technique also needs to be defined. Research as CSU may be able to provide more advanced four-dimensional data assimilation techniques in the future.

3.2.3 A subroutine will need to be written that will contain the data assimilation code and a procedure to access meteorological data.

3.2.4 The specific variables to be used for the data assimilation scheme, such as wind speed, wind direction, and temperature, need to be chosen.

3.2.5 As in task 3.1, a link to the NMC data base needs to be established for the fourdimensional data assimilation procedure. A procedure needs to be established to continually download and archive NMC data. This would require additional hardware and software development, and a link to the WCAL data base will have to be made.

3.2.6 Several simulations should be performed, with and without the final data assimilation technique, to determine the overall effectiveness on improvir.g mesoscale forecasts in the SRS area. The RAMS model should be able tc simulate mesoscale flow fields that vary in time, such as the passage of fronts, when the data assimilation technique is user.

3.2.7 Simulations must be performed to examine the numerical noise generated by the inclusion of the data assimilation method.

\subsection{Dispersion and Dosimetry}

4.1 Dispersion: The predicted wind field generated by the RAMS model will have to be coupled to the LPDM dispersion model to predict the transport of toxic chemicals or radionuclides.

\section{Lance O'Steen, Jerome Fast, Roger Pielke}

4.1.1 The LPDM dispersion model will need to be compiled and executed to test the type of inputs that are required and the type of output that is produced.

4.1.2 The wind field from one of the RAMS runs should be used to test the LPDM dispersion model so that the appropriate options are chosen in the dispersion model.

4.1.3 A link between the output of RAMS and the input to the dispersion model needs to be established.

4.1.4 The dispersion code will have to access the correct wind field forecast produced by the continuously-running atmospheric model.

4.1.5 A dense gas model may need to be developed for SRS emergency response applications. The RAMS code could be modified to include the interactions between the dense gas and the surrounding atmosphere. A much smaller grid spacing would be necessary to simulate dense gas releases. An evaluation of whether it is feasible to adapt RAMS to a dense gas application should be made.

4.1.6 New, more accurate procedures that represent dispersion, including the influences of covariances of the velocity components, need to be evaluated. 
4.2 Dosimetry: An independent program needs to be obtained or written that will convert the concentrations predicted by the LPDM model into effective dose rates.

\section{Lance O'Steen}

4.2.1 After the dispersion model has been run, a dosimetry model must be executed to compute the dose based on the concentrations predicted by the dispersion model.

4.2.2 Output from the dosimetry model needs to be archived for future graphical display.

\subsection{Operational Structure and System Integration}

5.1 Research and Development: Procedures need to be developed to get the RAMS and LPDM models running in a operational mode using the most efficient numerical and computational techniques.

Mobert Sharpley, Richard Ewing, Jerome Fast

5.1.1 The final atmospheric, dispersion, and dosimetry models that have been tested must be linked together in some manner.

5.1.2 The stability, accuracy, and robustness of RAMS codes will have to be examined to determine how well they will perform in an operational mode.

5.1.3 Numerically accurate and efficient nesting of the models via domain decomposition techniques will be used to numerically evaluate accurate and efficient methods of nesting the domain grids in the RAMS code.

5.1.4 The feasibility and benefits of implementing RAMS and LPDM codes in a multiprocessor environment will be assessed.

5.1.5 The overall execution time of the operational model could be reduced by eliminating unnecessary code or rewriting portions of the code to run more efficiently.

5.1.6 Most of the options that are required for the SRS application will be directly incorporated into the model or in its main input file. Once the modeling system has reached its final form, a menu could be set up to manage the model operation. This menu could contain a small number of options to be used for both the RAMS and LPDM models.

5.1.7 Operational versions of the advanced atmospheric/transport modeling system will be created after certain parameterization, boundary condition, initialization, data assimilation, dispersion, and dosimetry tasks have been completed.

5.1.8 Each operational version of the advanced atmospheric/transport modeling system should be evaluated to determine the performance in simulating toxic chemical or radionuclide releases at the SRS.

5.2 Graphical Output: The tabular output of the predicted variables by the RAMS code must be accessed by graphical programs to display the numerical results.

Jerome Fast, Lance O'Steen, SSESCO

5.2.1 The RAMS and LPDM codes have a companion program that produces graphical images of the numerical results produced by the atmospheric model. This graphic program needs to be compiled, once the NCAR graphics library is available on the Cray XMP computer and the IBM workstation. The graphic program can be tested with preliminary results from RAMS. 
5.2.2 The NCAR graphical program developed by ASTeR may need to be modified to meet the specific needs of the emergency response application. The default parameters may have to be altered.

5.2.3 Another companion program that produces graphical images of numerical results produced by RAMS and LPDM is being developed. This program needs to be evaluated and tailored to the SRS emergency response application.

5.2.4 The graphical images of concentration or dose contours will have to be overlaid on GIS (Geographic Information System) data for the emergency response application so that regions of dispersion can be related to population centers.

5.2.5 While graphical displays of atmospheric variables may not be necessary for the emergency response application, it is important that an option should be included in the resulting operational model to graphically overlay atmospheric and dispersion variables.

While there are many instances where a task cannot be initiated until another task completed, most of the tasks will be performed simultaneously by ETS and SCUREF researchers. It should also be noted that the number of tasks may change during the course of the project, depending on the outcome of some of the preliminary tests. This document will be updated periodically to reflect those changes.

\section{TARGET DATES}

It is expected that a preliminary operational version of the atmospheric/transport modeling system can be implemented before all of the necessary tasks have been completed. The newer versions of the modeling system will be tested to ensure that any modified code or additional numerical procedures improve the performance of the modeling system. The time period in which the tasks are completed will depend on the available computational resources as well as the schedules of ETS and SCUREF researchers (see Fig. 3). Several tentative dates have been set that establish reasonable goals in completion of the advanced atmospheric/transport modeling system.

\section{Version 1.0 8-1-92;}

- The RAMS and LPDM models will be executed on an IBM RS/6000 workstation.

- $\quad$ The RAMS and LPDM models will be executed on demand, but not on a routine basis.

- The RAMS model will incorporate the appropriate pararneterizations for the SRS area as indicated by tasks 1.4 and 1.5 .

- The RAMS model will use the default initial conditions and lateral boundary conditions contained in the version of the model.

- Both the RAMS and LPDM models will produce output files that may be archived.

- A procedure to graphically depict some of the results of the RAMS and LPDM models will be available.

\section{Version 2.0 2-1-93;}

- $\quad$ The RAMS model will be able to execute once or twice a day using simple shell-script commands to produce routine, real-time wind field forecasts for the SRS area.

- The RAMS model will incorporate time-dependent lateral boundary conditions obtained from a large-scale NMC forecast model using an existing procedure to increase the accuracy of the wind field forecasts.

- A vegetation parameterization and a second-order turbulence closure parameterization will be added to the model from software updates obtained from ASTeR. 


\section{Version 3.0 8-1-93:}

- A four-dimensional data assimilation procedure that will employ meteorological data from the NWS and SRS meteorological towers will be incorporated into the RAMS model to improve wind field forecasts in the SRS area.

- New hardware upgrades and optimizations the RAMS and LPDM codes will allow the modeling system to produce a complex dispersion forecast in a relatively short period of time.

- An elaborate dosimetry model will be added as a component of the advanced atmospheric/transport modeling system to produce dose information for SRS emergency response needs.

\section{Version 4.0 2-1-24:}

- Compurer code developed by SCUREF scientists (Shun Der Ko, Chris Evans, Robert Sharpley, Richard Ewing, and Roger Pielke) as a result of research for tasks 2.0 - 5.0 will be incorporated into the advanced atmospheric/transport modeling system to improve the initial conditions, boundary conditions, grid nesting methods, and numerical procedures.

\section{Yersion 5.0 8-1-94:}

- Two workstations will be devoted to the operation of the advanced atmospheric/transport modeling system to ensure that the models execute quickly for the emergency response application.

- The RAMS model will be rurning continuously (several cycles per day) on one workstation.

- The RAMS workstation designared will be connected to the WCAL data base and NWS products so that the four-dimensional data assimilation procedure ran "cess current meteorological data.

- The LPDM model will be executed on another workstation that is connected via ethernet to the RAMS workstation so that the dispersion model can access the latest wind field forecast.

- The advanced atmospheric/transport modeling system will execute the LPDM model, generate numerical results, and graphically depict the concentration of toxic chemicals or dose from radionuclides.

- The LPDM model will be executed on demand with source data and input parameters entered either by a user through a menu or automatically from existing databases.

- The LPDM model will have default SRS sites for release locations; however, an option will exist that will allow the user to choose the release location at any point within the domain.

- The graphical and tabular output of the modeling system will be directed to several terminals via ethernet.

- The total time to execute the modeling system will be short enough so that it may be used in an emergency response situation.

The preliminary version of the advanced atmospheric/transport modeling system, version 1.0, should be completed during the summer of 1992. The final version of the atmospheric/transport modeling system is planned to be in operation by the summer of 1994; however, the specific components in versions 2.0 - 4.0 may change depending upon the results of research performed for tasks $1.0-5.0$ during the next four years. 


\section{CONCLUSION}

Relatively simple models have been used by the emergency response organizations of SRS to predict accidental releases of toxic chemicals or radionuclides. These models have been shown to work reasonably well for simple atmospheric conditions, such as well-mixed boundary layers with moderate, constant winds. However, if strong horizontal or vertical variations in the wind were to occur over the SRS area, these models may produce highly unrealistic concentration or dose estimates. Strong horizontal or vertical variations in the wind in the SRS area are commonly caused by the passages of synoptic fronts or severe weather. The RAMS atmospheric model is better suited to simulate these wind events; therefore, the resulting dispersion can be predicted with a higher degree of accuracy.

This four-year project is designed to produce a state-of-the-art product for the emergency response organizations at SRS. It is expected to greatly improve the accuracy and reliability of dispersion forecasts in the region surrounding the SRS. Prognostic mesoscale atmospheric models are currently being developed to execute on an operational basis at a few organizations in the U. S., but as far we know, this is the first such advanced modeling capability designed srecifically for emergency response purposes. 


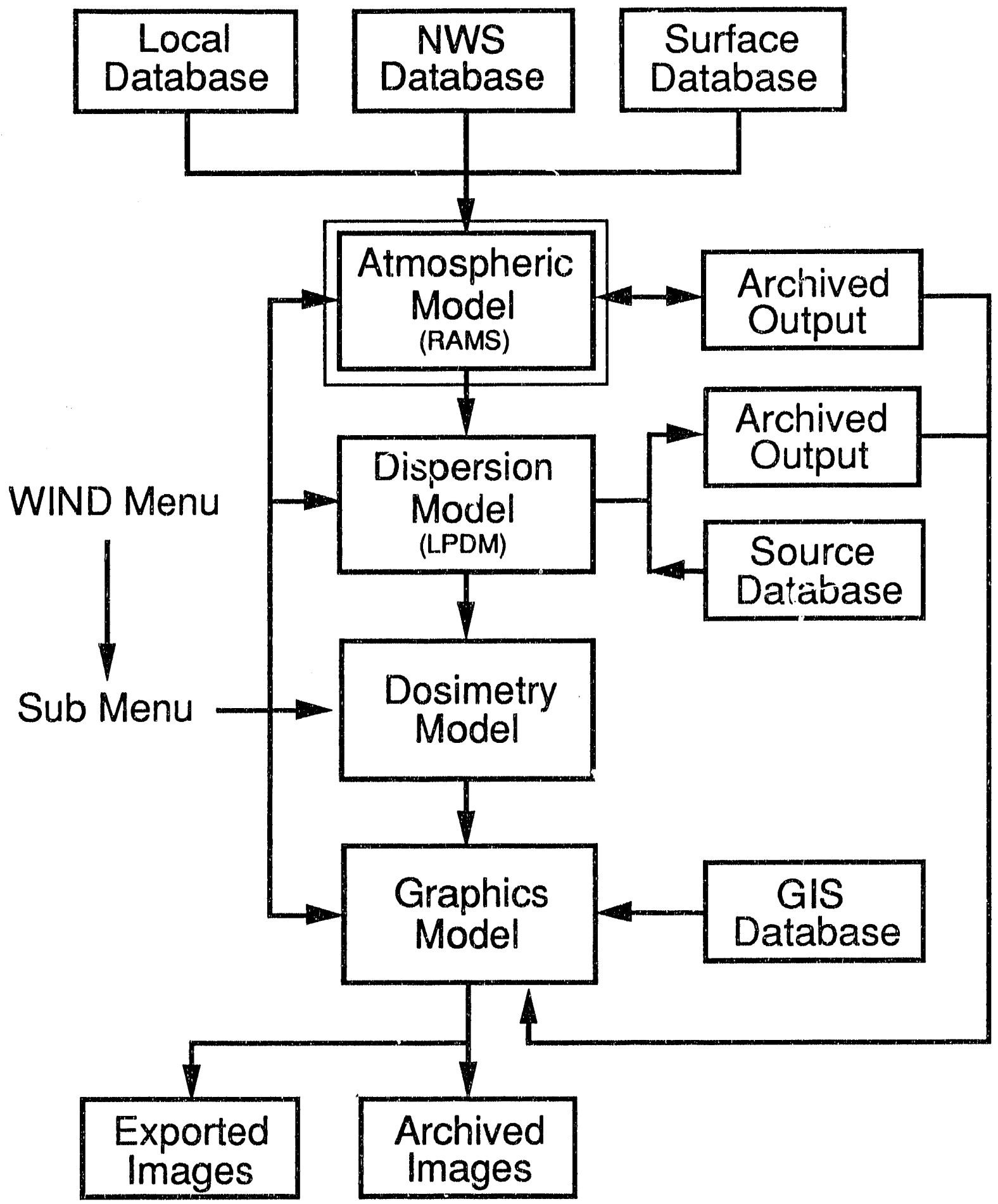

Fig. 1. General structure of proposed operational version of RAMS and LPDM models within framework of the WIND system menu. The RAMS atmospheric model executes continuously in the background (unavailable to the normal user). The user may select the LPDM dispersion model from the WIND system menu. The dispersion model automatically accesses the forecasted wind field produced by RAMS to predict the transport of toxic or radioactive materials in the SRS area. 


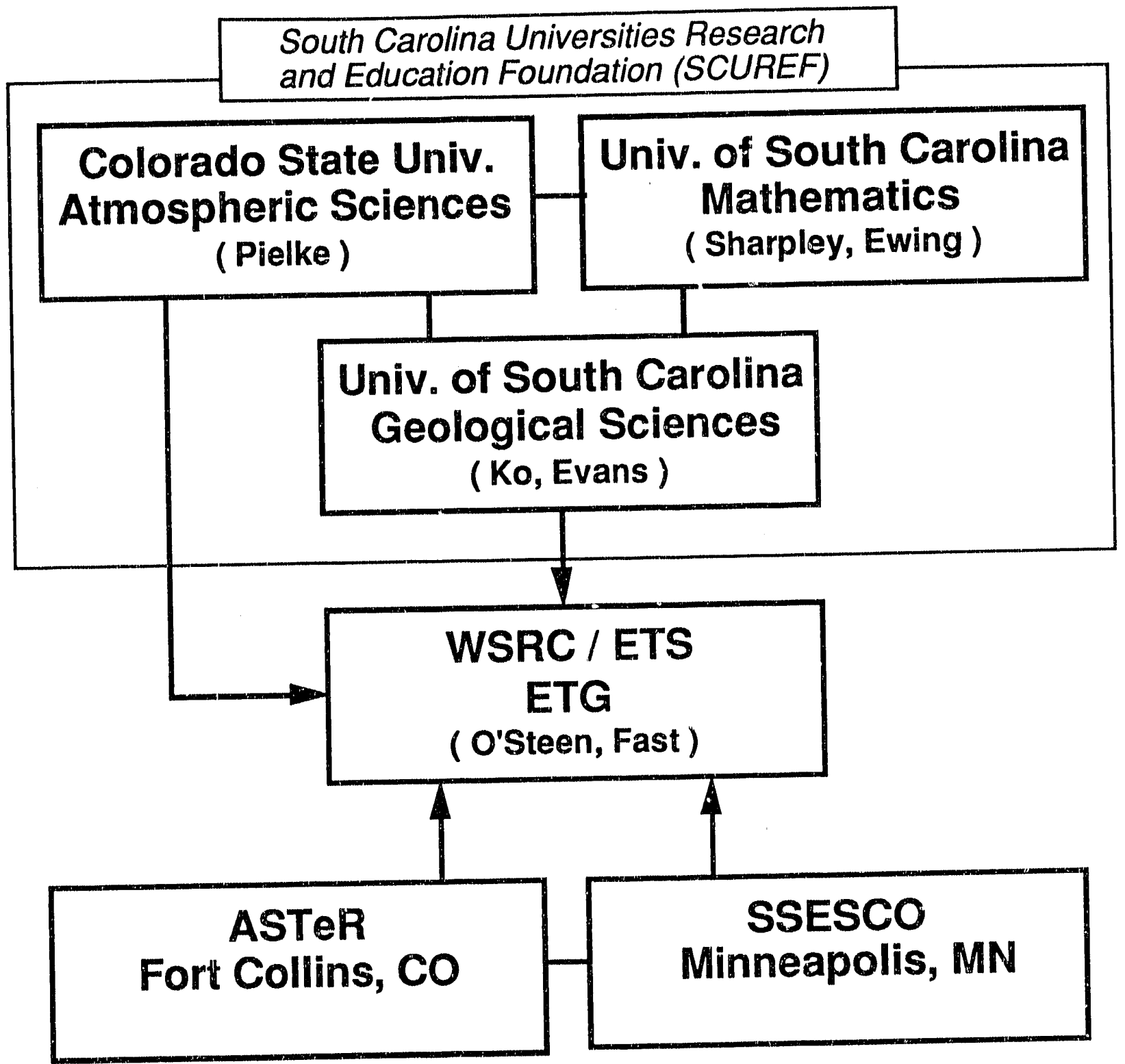

Fig. 2. Groups contributing to the development of the advanced atmospheric/transport modeling system. 


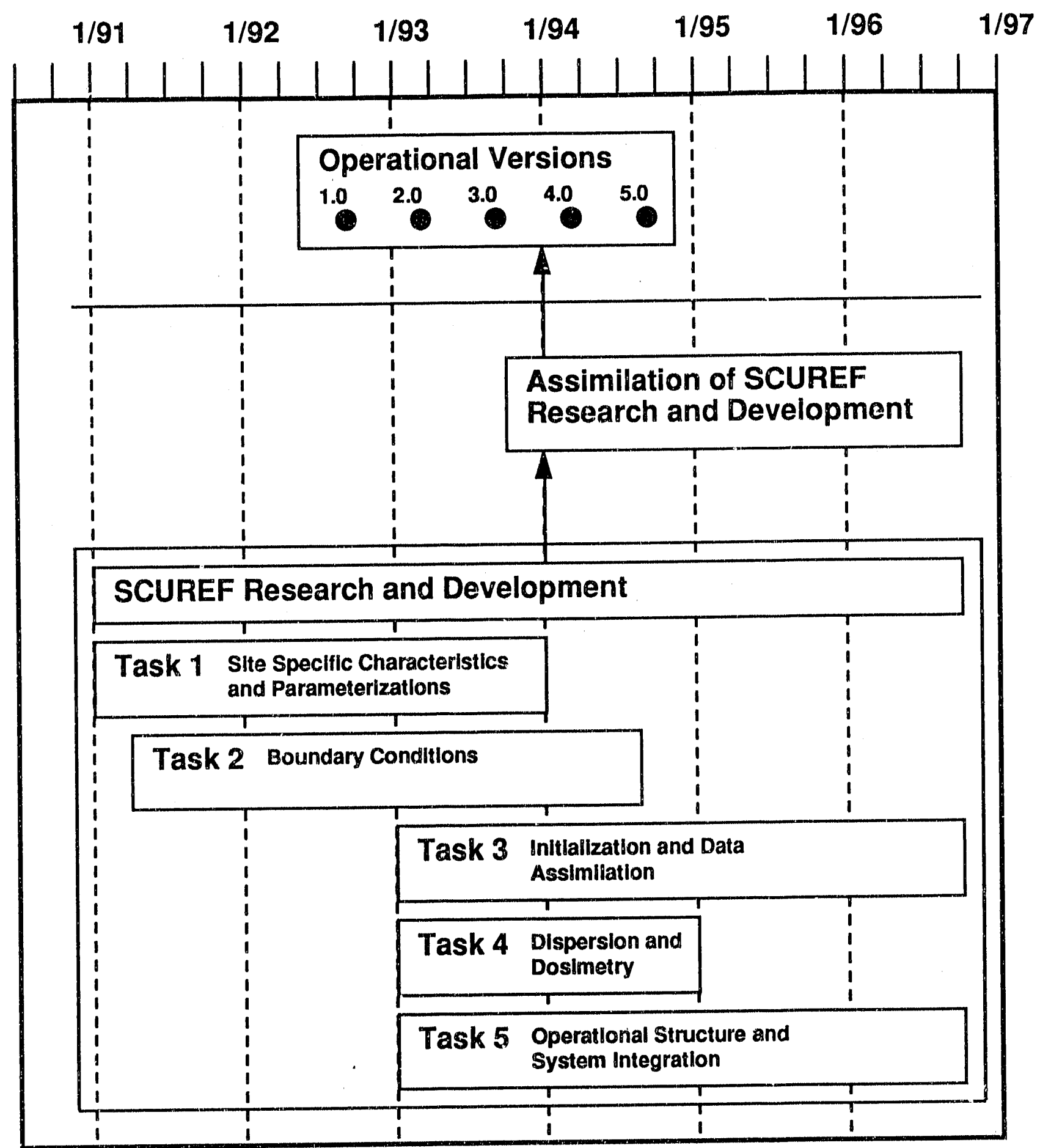

Fig. 3 Timeline depicting the tentative schedule for the tasks necessary to complete the advanced atmospheric/transpo: modeling system. 

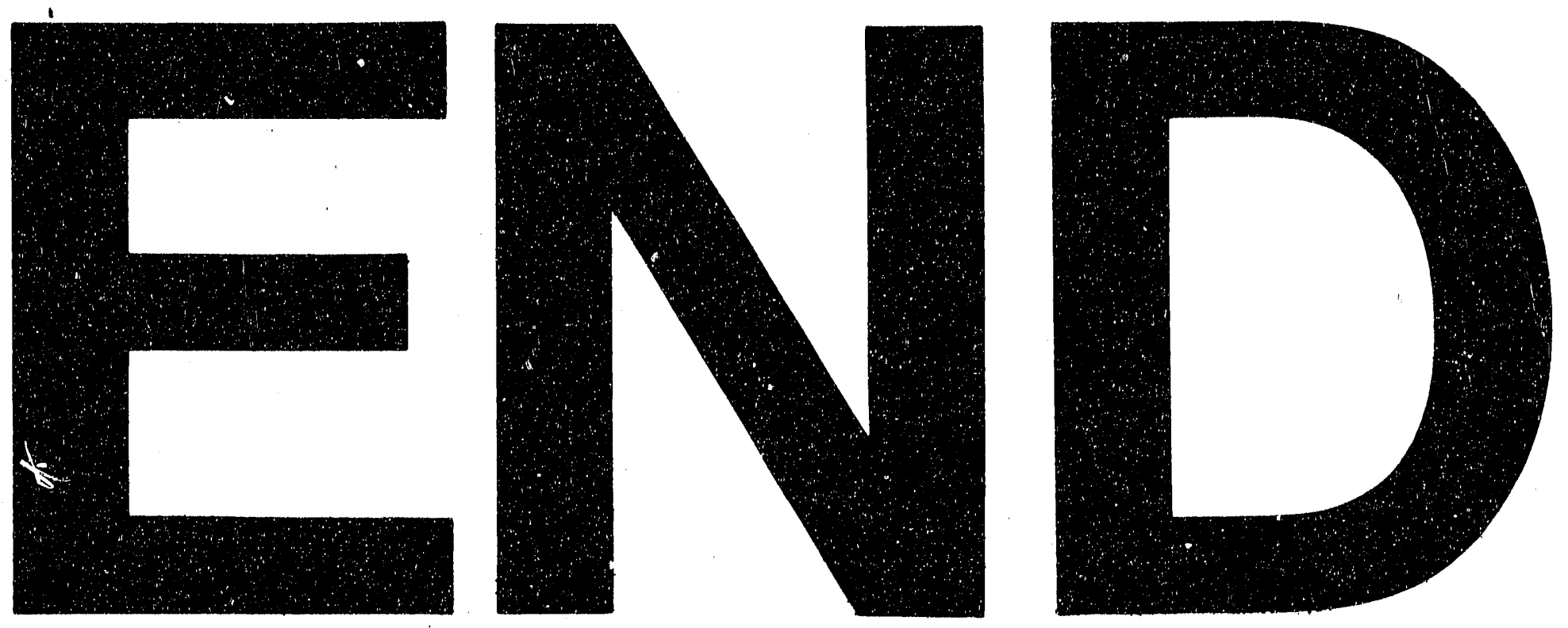

4
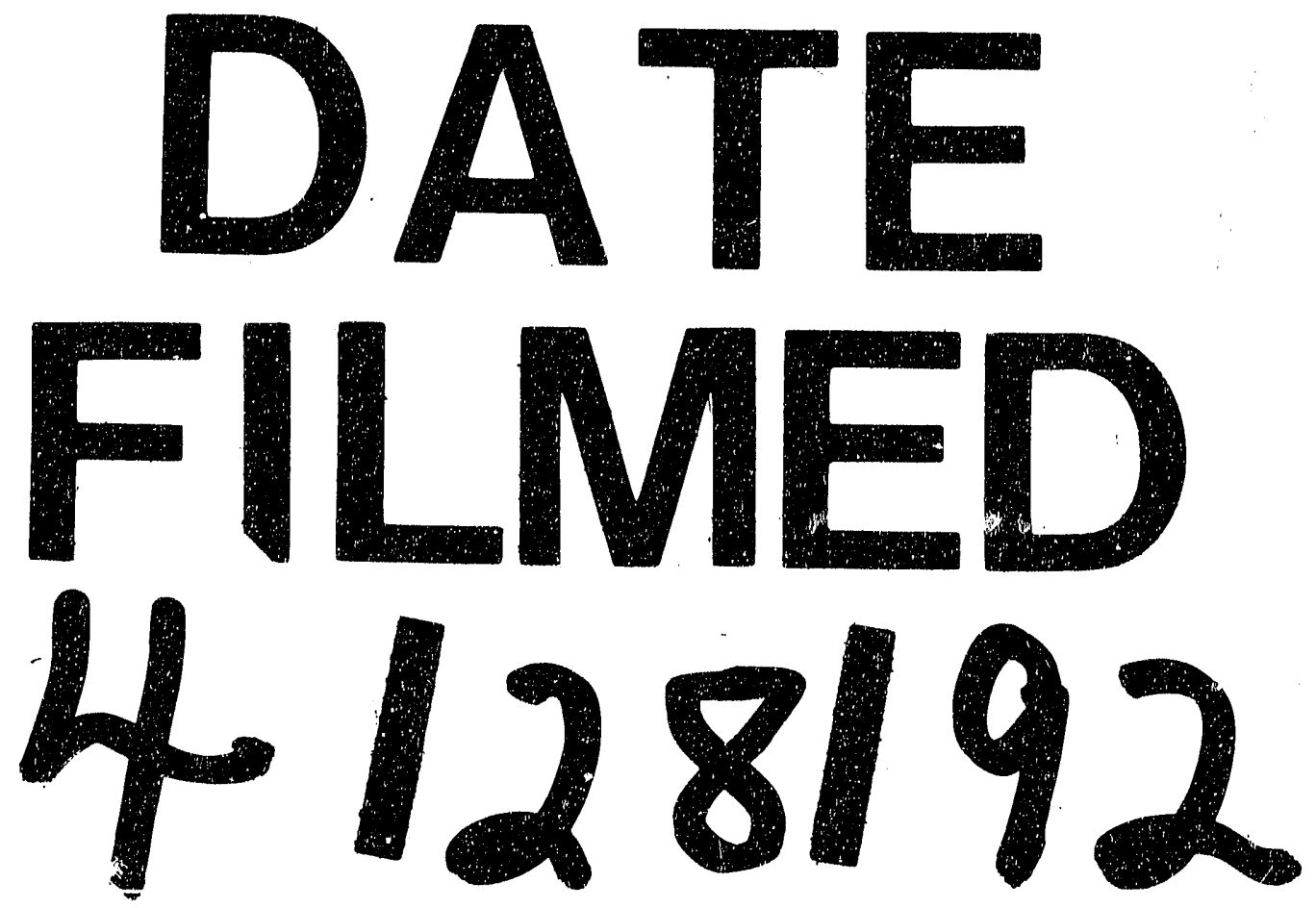


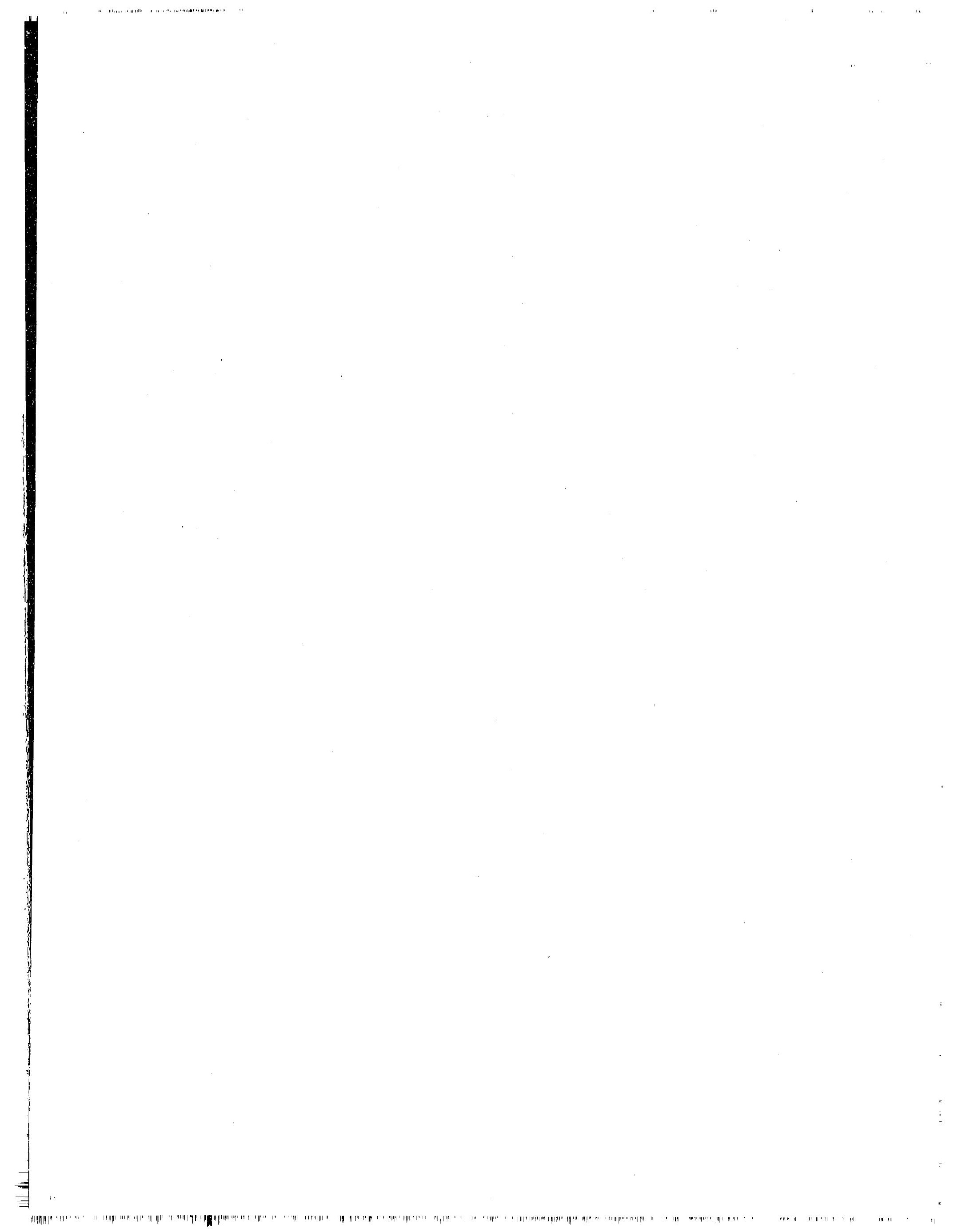

